\title{
Weakness of head turning in hemiplegia: a quantitative study
}

\author{
FL MASTAGLIA, W KNEZEVIC, PD THOMPSON \\ From the Department of Neurology, Queen Elizabeth II Medical Centre, Perth, Western Australia, Australia
}

SUMMARY The strength of head turning was measured in normal subjects and in patients with hemiplegia of vascular origin. In normals the strength of head turning to the two sides was not significantly different. In most patients with a cortical, capsular or high brainstem lesion the strength of head turning away from the side of the lesion was reduced. In patients with a medullary lesion the strength of head turning towards the side of the lesion was reduced. The significance of these findings is discussed in relation to the cortical control of the sternocleidomastoid muscle and other muscles involved in the movement of head turning.

Head turning movements are brought about by the ipsilateral splenius and contralateral sternocleidomastoid muscles acting as agonist pairs. ${ }^{1}$ As each cerebral hemisphere directs contralateral head turning, the action of the sternocleidomastoid muscle during this movement is thought to be under the control of the ipsilateral motor cortex. ${ }^{23}$ The neuroanatomical basis for this remains uncertain and it has been variably suggested that the cortical projection to the sternocleidomastoid motoneurons is undecussated $^{2}$ or doubly decussated. ${ }^{3}$ Weakness of head turning towards the side of a hemiplegia of cerebral origin was noted by Beevor ${ }^{4}$ in 1909 and is well recognised in patients with destructive lesions of the cerebral hemispheres. ${ }^{256}$ However, there has been no systematic quantitative study of this phenomenon. The aims of this study were to determine: (1) whether there are significant differences in the strength of head turning to the two sides in normal subjects; (2) how frequent the finding of weakness of head turning is in patients with hemiplegia of vascular origin and whether it persists beyond the acute stage; (3) whether observations on the strength of head turning in patients with brainstem lesions can shed any light upon the anatomical

Address for reprint requests: Professor FL Mastaglia, Department of Neurology, Queen Elizabeth II Medical Centre, Nedlands, W.A. 6009 , Australia.

Received 8 August 1984 and in final revised form 30 May 1985 Accepted 12 June 1985 course of the cortical pathway controlling the sternocleidomastoid.

\section{Methods}

Forty patients (27 males, 13 females; age range 32-81 years; mean age $62 \cdot 2$ years) with hemiparesis were studied 3 weeks to 6 months after the occurrence of a cerebrovascular lesion. Twenty-four had a right hemiparesis and 16 a left hemiparesis. The degree of weakness varied in different patients; most were unable to maintain elevation of the affected limbs against gravity and had severe loss of finger and hand dexterity. Twenty-two patients had an associated sensory deficit and eight had a hemianopia. None had anosognosia or severe neglect of the affected limbs. Patients who were confused or who could not cooperate fully in the testing procedure were excluded.

In most patients the responsible lesion (cerebral haemorrhage or infarction) had been confirmed by CT scanning. Thirty-six had a cortical or capsular lesion of the contralateral cerebral hemisphere; four had unilateral brainstem lesions, three in the midbrain or upper pons and the other in the medulla. Strength of head turning was measured to both sides using a Hammersmith Myometer. ${ }^{7}$ After initial instruction and a number of trials, three measurements of the maximal strength of head turning to each side were taken and the mean of the three was calculated. When one of the three values differed from the other two by more than 20 Newtons (N), (that is, was clearly submaximal), it was rejected and a further measurement taken. Identical measurements were made in 40 normal subjects ( 22 males, 18 females; age range 21-81 years; mean age 61 years). 


\section{Results}

The strength of head turning in the normal subjects ranged from 87 to $200 \mathrm{~N}$ to the right, and 92 to $187 \mathrm{~N}$ to the left; the mean values for right and left head turning were not significantly different in the whole group or in dextrals or sinistrals (table). The interside difference in the strength of head turning ranged from $-8.9 \mathrm{~N}$ to $+11.0 \mathrm{~N}$ (mean 1.6, SD 5.2); this was not statistically significant in the group as a whole or in the subgroup of right-handed subjects $(p>0.5$; Student's paired $t$ test).

In the patients with right hemiparesis the mean strength of head turning to the right was significantly lower than to the left (table) and the mean interside difference was significantly increased (mean 30.6, SD $14.5, \mathrm{p}<0.01)$. In the patients with left hemiparesis, the mean strength of head turning to the left was significantly lower than to the right (table) and the mean interside difference was significantly increased (mean - 16.0, SD 25.4, p < 0.02).

All but five of the 40 patients with hemiparesis had weakness of head turning to one side as shown by the interside strength of head turning difference of $20 \mathrm{~N}$ or greater. Of the 24 patients with right hemiparesis, $22 \mathrm{had}$ weakness of head turning to the right and two had equal strength of head turning to the two sides. Eleven of the 16 patients with left hemiparesis had weakness of head turning to the left (including three with right sided high brainstem lesions) and three had weakness of head turning to the right (including one with right sided medullary lesion). Two additional patients with unilateral medullary infarction due to vertebral artery occlusion also had weakness of head turning towards the side of the lesion.

\section{Discussion}

Our findings indicate that there are no significant differences in the strength of head turning to the two sides or correlation between strength of head turning and handedness in normal subjects. The majority of patients with hemiparesis due to a lesion of the dominant or non-dominant cerebral hemisphere have weakness of head turning away from the side of the lesion (that is of the sternocleidomastoid ipsilateral to the lesion) which may be detected even several months after an acute vascular lesion. This finding lends support to the concept of ipsilateral cortical control of the sternocleidomastoid in the movement of head turning.

It has been suggested that the corticospinal projection to the sternocleidomastoid motoneurons undergoes a dual decussation. ${ }^{3}$ The first decussation of the motor tracts concerned with head turning appears to take place in the pons ${ }^{8}$ while a second is postulated in the spinal cord below the level of $\mathrm{Cl} .^{9}$
Table Mean strength of head turning (SHT) to the right $(R)$ and left $(L)$ in normal subjects and patients with hemiparesis.*

\begin{tabular}{llll}
\hline & $S H T$ to $R$ & $S H T$ to L & $p \ddagger$ \\
\hline $\begin{array}{l}\text { Normals } \\
\text { Whole Group } \\
(40)\end{array}$ & $146.0 \pm 26.8+$ & $145.0 \pm 26.4$ & $>0.5$ \\
$\begin{array}{l}\text { Dextrals } \\
(35)\end{array}$ & $147.0 \pm 27.6$ & $146.0 \pm 27.0$ & $>0.5$ \\
$\begin{array}{l}\text { Sinistrals } \\
(5)\end{array}$ & $137.4 \pm 20.3$ & $135.8 \pm 20.8$ & $>0.5$ \\
$\begin{array}{l}\text { Hemiparesis } \\
\text { R. hemiparesis } \\
(24)\end{array}$ & $109.7 \pm 32.7$ & $136.2 \pm 26.3$ & $<0.01$ \\
L. hemiparesis & $130.8 \pm 24.0$ & $111.5 \pm 29.3$ & $<0.01$ \\
$\quad(16)$ & & & \\
\hline
\end{tabular}

*Values expressed as mean \pm standard deviation †Values in Newtons (1 Newton $=1 \mathrm{Kg} \cdot \mathrm{m} \cdot \mathrm{sec}^{-2}$ ).

$\ddagger$ Calculated using Student's $t$ test.

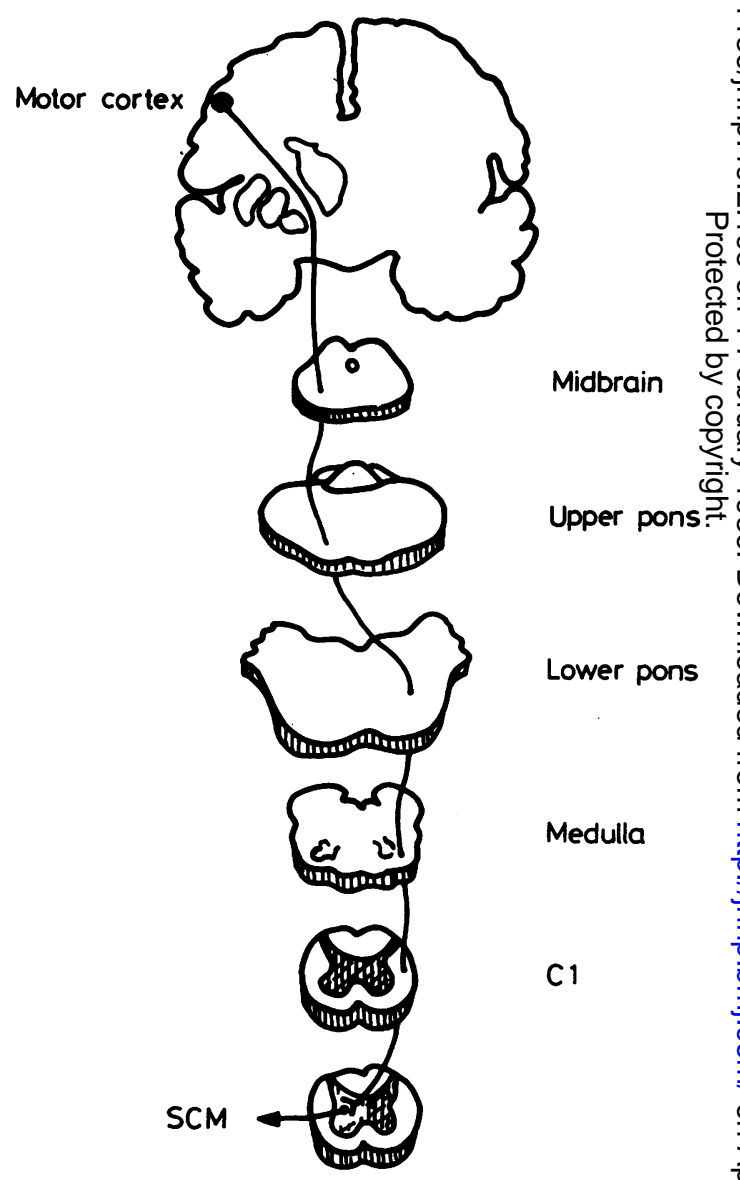

Fig Schematic representation of the putative descending pathway taken by corticospinal fibres to the sternocleidomastoid motor nucleus. For further details, see text. 
Our findings of weakness of contraversive head turning, suggesting paresis of the ipsilateral sternocleidomastoid, in patients with hemispheric or high brainstem lesions, and of ipsiversive head turning, implying paresis of the contralateral sternocleidomastoid, in patients with medullary lesions, supports the concept of such a dual decussation (fig). It is also possible that weakness of the posterior neck muscles contributed to the weakness of head turning in these lesions. The different patterns of weakness observed would be consistent with a single pontine decussation of the splenius upper motor neurones. Thus in hemispheric and high brainstem lesions, paresis of the contralateral splenius might contribute to the weakness of head turning away from the side of the lesion, while lesions of the medulla might produce paresis of the ipsilateral splenius and weakness of head turning towards the side of the lesion.

The explanation for the finding of weakness of head turning towards the side of the lesion in two patients with right hemisphere lesions is uncertain. On theoretical grounds this could occur if in such cases the left sternocleidomastoid were controlled indirectly by the left motor cortex via the corpus callosum and right cerebral hemisphere as suggested by Geschwind. ${ }^{3}$ Alternatively, it could be that in such cases the cortical control of the sternocleidomastoid is crossed as in the case of the limb muscles.

\section{References}

${ }^{1}$ Zangemeister WH, Stark L, Meienberg O, Waite T. Neural control of head rotation: electromyographic evidence. J Neurol Sci 1982;55:1-14.

${ }^{2}$ Balagura S, Katz RG. Undecussated innervation to the sternocleidomastoid muscle: a reinstatement. Ann Neurol 1980;7:84-5.

${ }^{3}$ Geschwind N. Nature of the decussated innervation of the sternocleidomastoid muscle. Ann Neurol 1981;10:495.

${ }^{4}$ Beevor CE. Paralysis of the movements of the trunk in hemiplegia. Br Med J 1909;1:881-5.

${ }^{5}$ Brain WR, Walton JN. Diseases of the Nervous System. 7th edition, London, Oxford University Press 1969 p. 21.

${ }^{6}$ Meyers IL. Conjugate deviation of the head and eyes. Its value in the diagnosis and localisation of abscess of the brain. Arch Otolaryngol 1931;13:683-708.

${ }^{7}$ Edwards RHT, McDonnell M. Hand-held dynamometer for evaluating voluntary-muscle function. Lancet 1974;ii:757-8.

${ }^{8}$ Bender MB, Schanzer S, Wagman IH. On the physiologic decussation concerned with head turning. Confinia Neurologica, 1964;24:169-81.

9 Iannone AM, Gerber AM. Brown-Sequard syndrome with paralysis of head turning. Ann Neurol 1982;12:116. 